\title{
Identifying latent classes of parents of children with hematological malignancies with respect to caregiving ability: a latent class analysis
}

\author{
Jingting Wang ${ }^{1}$, Xuanyi Bi ${ }^{1}$, Jichuan Wang ${ }^{2}$, Xianlan Zheng ${ }^{3}$, Yingwen Wang ${ }^{4}$, Huifang \\ $\mathrm{Wu}^{5}$, Qi Yang ${ }^{5}$, Fang Liu ${ }^{4}$, Linyu $\mathrm{Ma}^{3}$, and Changrong Yuan ${ }^{6}$ \\ ${ }^{1}$ Naval Medical University \\ ${ }^{2}$ Children's National Medical Center \\ ${ }^{3}$ Children's Hospital of Chongqing Medical University \\ ${ }^{4}$ Children's Hospital of Fudan University \\ ${ }^{5}$ Children's Hospital of Soochow University \\ ${ }^{6}$ Fudan University
}

September 24, 2020

\begin{abstract}
Objectives: This study aimed to identify unobserved subgroups of parents of children with hematological malignancies with respect to caregiving ability and examine the associations of the latent class membership with individual characteristics. Methods: A total of 392 parents of children with hematological malignancies in China were surveyed with the Hematologic Malignancies' Family Caregiver Skills Scale and a study-specific demographic information questionnaire. Latent class analysis(LCA) was applied to identify latent classes of parents based on caregiving ability measures. Multinomial logistic regression model was used to investigate the associations of socio-demographic and clinical characteristics with the latent class membership. Results: Results from the LCA suggested a 3-class solution: Class 1- "high caregiving ability" class(n=131, 33.4\%), Class 2- "medium caregiving ability" class $(\mathrm{n}=170,43.4 \%)$, and Class 3- "low caregiving ability" class $(\mathrm{n}=91,23.2 \%)$. Socio-demographic and clinical characteristics, such as having lower level of education, being married, having higher household income, more daily caregiving time, and having older children, as well as children not diagnosed with leukemia, had significantly larger odds of being classified in Class 1 than in Class 3. When comparing Class 2 with Class 3, the findings remained basically unchanged except that the effect of gender of parent became statistically significant, and the effect of diagnosis of children became insignificant. Conclusion: There existed three distinct priori unknown classes of parents of children with hematological malignancies in regard to caregiving ability. The class membership was significantly associated with parent's socio-demographics and child's clinical measures. These findings may be helpful in the development of personalized caregiving ability interventions.
\end{abstract}

\section{Introduction}

The incidence and mortality of childhood cancer have increased annually over the past decade, making it surpass accidental trauma as the leading cause of childhood death in 201611Bray F, Ferlay J, Soerjomataram I, et al. Global cancer statistics 2018: GLOBOCAN estimates of incidence and mortality worldwide for 36 cancers in 185 countries. CA Cancer J Clin.2018; 68(6):394-424.-22Kyu HH, Stein CE, Boschi Pinto C, et al. Causes of death among children aged 5-14 years in the WHO European Region: a systematic analysis for the Global Burden of Disease Study 2016. Lancet Child Adolesc Health.2018; 2(5):321-337.. The overall annual incidence of childhood cancer varied between 46 and 165 per million33Rodriguez GC, Friedrich P, Alcasabas $\mathrm{P}$, et al. Toward the cure of all children with cancer through collaborative efforts: pediatric oncology as a 
global challenge. J Clin Onco, 2015; 33(27): 3065-3073.. Hematological malignancy is the most common cancer among children younger than 15 years old44World Cancer Report: Cancer Research for Cancer Prevention. International Agency for Research on Cancer Publications Website. https://publications.iarc.fr/586. Updated Feb 4, 2020. Accessed Sep 16, 2020. However, with the advances in treatment over the past decade, the 5-year and 10-year survival rates for childhood cancer are more than $80 \%$ in high-income countries55Dixon SB, Bjornard KL, Alberts NM. Factors influencing risk-based care of the childhood cancer survivor in the 21st century. CA Cancer J Clin. 2018; 68(2):133-152.66Bhakta N, Force LM, Allemani C, et al. Childhood cancer burden: a review of global estimates. Lancet Oncol. 2019; 20(1): e42-e53.77Ward ZJ, Yeh JM, Bhakta N, et al. Global childhood cancer survival estimates and priority-setting: a simulation-based analysis. Lancet Oncol. 2019; 20(7):972-983. Based on current and long-term survival rates, the number of childhood cancer survivors is expected to reach 500,000 by 202088Robison LL, Hudson MM. Survivors of childhood and adolescent cancer: life-long risks and responsibilities. Nat Rev Cancer. 2014; 14(1):61-70..

Despite the better survival rates, the diagnosis of hematological malignancy is undoubtedly a heavy blow to the children and their families. Due to the complexity and particularity of hematological malignancy, children are suffering from a series of discomforts caused by the disease and the long-term chemotherapy99Abedin S, Altman JK. Acute promyelocytic leukemia: preventing early complications and late toxicities. Hematology Am Soc Hematol Educ Program. 2016; 2016(1):10-15.'1010Berger AM, Mitchell SA, Jacobsen PB, et al. Screening, evaluation, and management of cancer-related fatigue: Ready for implementation to practice? CA Cancer J Clin. 2015; 65(3):190-211.. As the primary caregivers, the parents need to participate in children's physical and mental support and all-round care throughout the diagnosis and treatment1111Wang JT, Shen NP, Zhang XY, et al. Care burden and its predictive factors in parents of newly diagnosed children with acute lymphoblastic leukemia in academic hospitals in China. Support Care Cancer.2017; 25(12):37033713.. However, caring for children with hematological malignancies is a rare and challenging experience for parents without medical training, and most parents are seeking professional assistance to improve their caregiving ability1212Wang JT, Yao NL, Wang YY, et al. Developing "Care Assistant": A smartphone application to support caregivers of children with acute lymphoblastic leukemia. J Telemed Telecare.2016; 22(3):163-171.'1313Wang JT, Howell D, Shen NP, et al. mHealth Supportive Care Intervention for Parents of Children with Acute Lymphoblastic Leukemia: Quasi-Experimental Pre- and Postdesign Study. JMIR Mhealth Uhealth. 2018; 6(11):e195..

Caregiving ability refers to the knowledge and skills that the caregiver has acquired to supply the patient what he or she needs1414Clarke DJ, Hawkins R, Sadler E, et al. Introducing structured caregiver training in stroke care: findings from the TRACS process evaluation study. BMJ Open. 2014: 4(4):e004473.. Parents' caregiving ability directly affects the rehabilitation process and the prognosis of their children1515Aburn G, Gott M. Education given to parents of children newly diagnosed with acute lymphoblastic leukemia: a narrative review. J Pediatr Oncol Nurs. 2011; 28:300-305.. However, existing studies mostly focused on caregivers of adult patients with cancer and aimed at improving their caregiving ability1616Mazanec SR, Sandstrom K, Coletta D, et al. Building Family Caregiver Skills Using a Simulation-Based Intervention: A Randomized Pilot Trial. Oncol Nurs Forum. 2019; 46(4):419-427.'1717Potter P, Olsen S, Kuhrik M, et al. A DVD program on fall prevention skills training for cancer family caregivers. J Cancer Educ. 2012; 27(1):83-90.. Studies on caregiving ability in parents of children newly diagnosed with leukemia in China have showed that parent's caregiving ability was at a low level1818Wu HF, Bi XY, Li J, et al. Analysis on care ability and its influencing factors for parents of children with leukemia. Nurs J Chin PLA. 2020; 37(2):18-22.. However, the findings were based on average statistics and did not necessarily mean that all the parents of children with hematological malignancies have a low level of caregiving ability. Importantly, the statistical analyses conducted in those studies assumed the patents were a homogeneous population regarding caregiving abilities. Thus, the statistical results in the studies might be biased. ${ }^{18,1919 S h e n ~ M, ~ L i a n g ~ P R, ~}$ Ma JL, et al. Influencing factors of caregiving ability of family caregivers of leukemic children. Modern Clinical Nursing. 2018; 17(9):50-55.'2020Mao ZQ, Xiao RT, Yang L, et al. Investigation on the influence of caregivers' care ability on children with leukemia. Today nurse. 2018; 25(6):43-45.. In order to better understand the populations, the present study used latent class analysis (LCA) to test the heterogeneity of 
this special population, i.e., parents with children with hematological malignancies, identify the unobserved distinct sub-populations/latent classes in the target population, and examine the characteristics of parents in different classes. The study armed to provide useful implications for development of a targeted and group-centered intervention for parents to improve their caregiving ability.

Latent class analysis (LCA) is an appropriate method to classify parents according to their caregiving ability, considering population heterogeneity. Based on the responses on explicit test questions LCA identifies the optimal number of latent classes of parents and the prevalence of each class2121Miranda VPN, Dos Santos Amorim PR, Bastos RR, et al. Evaluation of lifestyle of female adolescents through latent class analysis approach. BMC Public Health.2019; 19(1):184.. Tillery et al used LCA to classify caregivers of childhood cancer survivors aged 10 to 18 , based on the parent-child relationship functioning. Their findings would help to improve psychological and social outcomes in young survivors of childhood cancer2222Tillery R, Willard VW, Howard Sharp KM, et al. Impact of the parent-child relationship on psychological and social resilience in pediatric cancer patients. Psychooncology. 2020; 29(2):339-346.. However, no existing studies used LCA to analyze the caregiving ability in parents of children with hematological malignancies.

In the present study, we used LCA to identify a priori unknown latent classes of parents of children with hematological malignancies based on caregiving ability measures. Once latent classes were identified, the multinomial logistic model was used to examine the effects of socio-demographic and clinic characteristics on latent class membership. The findings of this study will be helpful for studying disparities in parents' caregiving ability and provide targeted interventions to parents in the different caregiving ability subgroups.

\section{Methods and materials}

\section{Sample and data collection}

Participants were 392 parents of children with hematological malignancies recruited from three children's hospitals in Shanghai, Jiangsu, and Chongqing, China. The survey was conducted from May 2018 to April 2020. The eligible criteria for parents and children were as follows: (1) the child met the diagnostic criteria for hematological malignancy11Zhang ZN, Shen T. Diagnostic and therapeutic criteria of hematologic diseases. Beijing: Science Press; 2007. by bone marrow cytology, immunohistochemical and genetic analysis, or histochemical staining, (2) the child was under treatment and was less than 15 years old, (3) the child had no other serious or chronic diseases, (4) the parent had high school diplomas or higher education, and could communicate fluently in Mandarin, (5) the parent took the major responsibility for the daily caregiving of the child, (6) the parent volunteered for the survey. This survey received ethical review approval from the Naval Medical University (NO.HJEC-2018-YF-001). The participants were informed of the aim of this study before the survey was conducted. No procedures were performed until participants signed the informed consent.

\section{Measures}

Information about the socio-demographic and clinical characteristics of parents and their children were collected in survey. Parents' caregiving ability was measured based on their last week's experience, using the Hematologic Malignancies' Family Caregiver Skills Scale (HMFCGSS) that was previously validated in hematologic malignancies' family caregivers in China (Cronbach's $\alpha=0.920$ )11Qian SJ. The adaptation of hematologic malignancies family caregiver skills scale. Master thesis. Soochow University, Suzhou, Jiangsu, China, 2016.. HMFCGSS consists of 6 domains with a total of 23 items: disease cognitive ability (DCA) with 3 items, general approaches to caregiving (GAC) with 3 items, person-centered care skills (PCS) with 5 items, emotional management ability (EMA) with 4 items, appraising supportive resources (ASR) with 3 items, balancing caregiving needs and one's own needs (BCN) with 5 items (see Appendix). Each of the 23 items was measured on a 5-point scale ranging from not difficult (1) to very difficult (5). In the present study, all the items were recoded as dichotomous indicator of parent's caregiving ability: 1-"no difficulty" (if item score [?]2) vs. 0-" at least some difficulty" (if item score[?]3).

\section{Data analysis}


The 23 dichotomous caregiving ability indicators were used for Latent Class Analysis (LCA)1125 Wang J, Wang X. Structural Equation Modeling: Applications Using Mplus, 2d Edition. New York: John Wiley; 2020.. The LCA analysis was started with a single-class solution and increased the number of classes (to four) while comparing model fit. Model fit statistics and indices include the Akaike Information Criterion (AIC), Bayesian Information Criterion (BIC), adjusted Bayesian Information Criterion (aBIC), Bootstrapped Likelihood Ratio Test (BLRT), Vuong-Lo-Mendell-Rubin likelihood ratio test (LMR), Lo-Mendell-Rubin adjusted LRT test(aLMR) and entropy score ${ }^{25}$. Lower values on AIC, BIC and aBIC indicate better model fit; significant p-values on the BLRT, LMR, and aLMR indicate the $\mathrm{k}$ class model is more preferable than the k-1 class model ${ }^{25}$. BIC and BLRT perform better than other fit statistics/indices in determining the number of latent classes. The entropy statistic measures the certainty of class classification. The values of entropy range from 0 to 1 , and a value closer to 1 indicating better classification ${ }^{25}$. For a LCA model with entropy [?]0.80, the latent class membership estimated from the model can be saved as "observed" categorical variables for further analysis22Clark SL. Mixture Modeling with Behavioral Data. Doctor thesis. University of California, Los Angeles, CA, 2010.. In the present study, we used a multinomial logistic regression model to examine the effects of demographic and clinical variables on the latent class membership. LCA was estimated using Mplus 7.0, and other analyses were conducted using SPSS 22.0. All tests of statistical significance were 2 -sided at $\alpha=0.05$ level.

\section{Results}

\section{Socio-demographic characteristics}

A total of 392 parents of children with hematological malignancies who completed the questionnaires were eligible for this survey. The socio-demographic characteristics of parents and children are described in Table 1.

\section{Latent class analysis}

Mode fit statistics/indices are shown in Table 2. The single-class model had the largest AIC, BIC, and ABIC, compared to all other models. In addition, the LR tests of the 2-class model were all statistically significant (LMR $P<0.0001$, aLMR $P<0.0001$, and BLRT $P<0.0001$ ), indicating the population is not homogeneous, but heterogeneous. Comparing the 2-class and 3-class models, the latter had smaller information criterion indices (AIC, BIC, and ABIC) and all significant LR tests (LMR $P=0.0021$, aLMR $P=0.0021$, and BLRT $P$ $<0.0001)$. We, therefore, rejected the 2 -class model in favor of 3 or more classes. Comparing the 3 -class and the 4-class models, BIC (9705.262), LMR $(P=0.7261)$, and aLMR $(P=0.7265)$ favored the 3 -class model, but AIC, aBIC, and BLRT favored the 4-class model. On the balance of model fit, model parsimony, and clinical interpretability of the results, the three-class model was selected. Figure 1 displays the results of the 3-class solution. The attribution probabilities of the model were $0.923,0.948$ and 0.958 , respectively, and entropy $=0.872$ indicating good quality of class classification. The plot of item response probabilities endorsing "no difficulty" for the 23 caregiving ability indicators in each of the classes shows explicit patterns of caregiving ability in the parents of children. The probabilities of endorsing "no difficulty" in Class 1 $(\mathrm{n}=131,33.4 \%)$ are all high across six domains. We, therefore, labeled this class as "high caregiving ability" class. The other two classes (Classes 2 and 3$)$ were labeled "medium caregiving ability" (n=170, 43.3\%) and "low caregiving ability" ( $\mathrm{n}=91,23.2 \%)$ classes, respectively, based on the item response probabilities in the classes.

\section{Analysis of caregiving ability by latent class}

Table 3 shows the mean scores of the 6 caregiving ability domains by latent classes. In each class, the GAC mean score was the lowest, compared to other domain scores. This indicated that parents all had higher level of ability of general caregiving on average. In Class 3 (the "low caregiving ability" class) and Class 2 (the "medium caregiving ability" class), the ASR score was higher than other domain scores, indicating that parents had lower ability of appraising supportive resources in the two classes. Although the DCA mean score in Class 1 (the "high caregiving ability" class) was lower than those in the other two classes, this score was much higher than other domain scores in Class 1, indicating that parents in this class also had some 
difficulty in disease cognitive.

\section{The multinomial logistic regression model}

Selected results of the multinomial logistic regression model are shown in Table 4. Parents with lower education (e.g., high school or junior college) and married status were more likely to be classified in the "high caregiving ability" class than in the "low caregiving ability" class. The corresponding ORs are: $\mathrm{OR}=2.679(95 \% \mathrm{CI} 1.116,6.430)$ for high school; OR=3.734 (95\%CI 1.473, 9.463) for junior college; and $\mathrm{OR}=4.298(95 \% \mathrm{CI} 1.070,17.256)$ for married parents (see the second column of Table 5). On the contrast, parents with the following characteristics were less likely to be in the "high caregiving ability" class, but in the low caregiving ability class: parents with average monthly household income of 2000-5000CNY (\$285$713)(\mathrm{OR}=0.245,95 \% \mathrm{CI} 0.087,0.691)$ or $5000-8000 \mathrm{CNY}(\$ 713-1411)(\mathrm{OR}=0.207,95 \% \mathrm{CI} 0.071,0.608)$, daily caregiving time of less than $12 \mathrm{~h}(\mathrm{OR}=0.308,95 \% \mathrm{CI} 0.140,0.675)$ or 12 to $18 \mathrm{~h}(\mathrm{OR}=0.293,95 \% \mathrm{CI} 0.115$, $0.750)$, children aged less than 3 years old $(\mathrm{OR}=0.137,95 \% \mathrm{CI} 0.053,0.355)$ and with a diagnosis of leukemia $(\mathrm{OR}=0.056,95 \% \mathrm{CI} 0.006,0.520)$.

Model results for comparing "medium caregiving ability" class with the "low caregiving ability" class are shown in the third column of Table 5. Parents who were married (OR=5.447, 95\%CI 1.588, 18.687), fathers $(\mathrm{OR}=2.399,95 \% \mathrm{CI} 1.162,4.956)$, had high school education $(\mathrm{OR}=3.032,95 \% \mathrm{CI} 1.348,6.818)$ or junior college $(\mathrm{OR}=5.416,95 \% \mathrm{CI} 2.269,12.930)$ were more likely to be classified in the "medium caregiving ability" class than in the "low caregiving ability" class. Parents with average monthly household income of 2000$5000 \mathrm{CNY}$ (\$285-713) (OR 0.245, 95\%CI 0.087, 0.691) or 5000-8000CNY (\$713-1411) (OR 0.207, 95\%CI $0.071,0.608$ ), daily caregiving time less than $12 \mathrm{~h}$ (OR $0.237,95 \% \mathrm{CI} 0.113,0.499$ ) or 12 to $18 \mathrm{~h}$ (OR 0.422 , $95 \%$ CI $0.185,0.965$ ), and children younger than 3 years old (OR $0.420,95 \%$ CI $0.180,0.981$ ) were more likely to be in the "low caregiving ability" class than in the "medium caregiving ability" class.

\section{Discussions}

\section{The latent classes of parents of children with hematological malignancies}

To the best of our knowledge, this is the first study that used the LCA to identify unobserved subpopulations/classes of parents of children with hematological malignancies in China. Parents in the study were classified into 3 distinct a priori unknown classes regarding to caregiving ability measured by HMFCGSS: Class 1-"high caregiving ability" class; Class 2-"medium caregiving ability" class; and Class 3-"low caregiving ability class". Class 1 accounted for $33.4 \%(\mathrm{n}=131)$ of the sample. The members in Class 1 had a medium level of DCA and high levels of the other five domains. Class 2 accounted for $43.4 \%(n=170)$ of the sample. Overall, the parents in Class 2 had a medium level of DCA, EMA, ASR and BCN, and relatively high levels of GAC and PCS. Class 3 accounted for $23.2 \%(n=91)$ of the sample. Parents in Class 3 had a medium level of GAC and low levels of all the other domains. For parents in the "low caregiving ability" class, caring for children with hematological malignancies was really challenging.

\section{Caregiving ability of parents in three latent classes}

As shown in Table 3, parents in all the 3 classes had a higher level of GAC than the other domains of caregiving ability, indicating that caregivers were more capable in general approaches of caregiving than other aspects of caregiving. These results showed that all parents had confidence in providing daily routine care and offering timely help when their children needed. In the "medium caregiving ability" class and "low caregiving ability" class, parents' lowest caregiving ability was ASR, which indicated that the most difficult part of taking care of their children in these two classes was to appraise supportive resources. While in the "high caregiving ability" class, caregivers' lowest caregiving ability is DCA, which indicated that the greatest difficulty in caregiving in this class was cognitive related, such as the assessment of the children's condition and the observation of changes in the children's condition, learning information on disease treatment and caring for the children, and contacting with the professional healthcare providers. The measurement tool, HMFCGSS, is a self-reported scale with six domains. A high total score of HMFCGSS may not necessarily mean that the parents have high caregiving ability in all the aspects. Professional healthcare providers need 
to pay attention to whether the parents have low ability in any aspect of caregiving, and what targeted support need to be provided to the parents.

\section{Association between socio-demographic and clinic characteristics and latent class membership}

While fathers of the children in the study were more likely to be in the "medium caregiving ability" class, mothers were more likely to be in the "low caregiving ability" class. In literature, mothers are more often than fathers to take care of sick children and take children to hospital11Yayan EH, Düken ME. A comparison of caregiving burden and social support levels of parents of children undergoing liver transplant. J Pediatr Nurs. 2019; 47:e45-e50.. In Chinese traditional culture, mothers are expected to be the primary caregivers for children in family and in general provide more practical daily care and emotional care for sick children. In our study, 281 mothers (71.7\%) took the major responsibility of the daily caregiving of their sick children. In a tragic situation when a child of a family is diagnosed with cancer, it would be very likely for the mother, the primary caregiver, to confront with more new challenges in caregiving for the sick child. These new challenges might lead to mothers' feeling of low caregiving ability.

Divorced, widowed or separated parents were more likely to be in the "low caregiving ability" class. Studies indicated that married parents had more access to social support, especially for the support from their partner, and tended to report lower caregiving burden22Arab M, Bernstein C, Haghshenas A, et al. Factors associated with caregiver burden for mothers of children undergoing Acute Lymphocytic Leukemia (ALL) treatment. Palliat Support Care.2019; 1-8.'33Wiener L, Viola A, Kearney J, et al. Impact of caregiving for a child with cancer on parental health behaviors, relationship quality, and spiritual faith: do lone parents fare worse? J Pediatr Oncol Nurs. 2016; 33(5):378-386. In addition, married parents usually have less financial burden caused by the children's disease treatment, so they could concentrate on taking care of their children, which could help to improve their caregiving ability44Granek L, Nathan PC, Rosenberg-Yunger ZR, et al. Psychological factors impacting transition from paediatric to adult care by childhood cancer survivors. J Cancer Surviv. 2012; 6(3):260-269..

Parents who received lower education (high school or just junior college) were more likely to be classified in the "high caregiving ability" class. In the present study, almost half (46.7\%) of the sample (n=183) with lower education (high school or junior college) had more than one child. Therefore, they had more caregiving experience in taking care of their children. In contrary, parents with higher education (bachelor degree or above) were more likely to have a single child, more co-caregivers, and less daily caregiving time. This may explain their less caregiving experience and low caregiving ability.

Lower income had a significant effect on the likelihood of being in the "low caregiving ability" class. Parents confronted with a high medical cost for their child disease treatment, expenses of caregiving needs, and often change in employment status following a child's cancer diagnosis. All those might reduce the financial stability of the family55Tsimicalis A, Stevens B, Ungar WJ, et al. Shifting Priorities for the Survival of My Child: Managing Expenses, Increasing Debt, and Tapping Into Available Resources to Maintain the Financial Stability of the Family. Cancer Nurs.2020; 43(2):147-157.. Moreover, the financial burden on parents of children with hematological malignancies is much heavier than that of any other cancer66Pagano E, Baldi I, Mosso ML, et al. The economic burden of caregiving on families of children and adolescents with cancer: a population-based assessment. Pediatr Blood Cancer.2014; 61(6):1088-1093.. A study on caregivers of adolescents and young adults with complex medical conditions found that family income directly predicted family functioning and greatly contributed to caregivers' caregiving ability77Deatrick JA, Hobbie W, Ogle S. Competence in caregivers of adolescent and young adult childhood brain tumor survivors. Health Psychol.2014; 33(10):1103-1112. So medical insurance with a high reimbursement rate and more financial help for the parents are needed, which will also have positive effect on improving parents' caregiving ability.

Parents with shorter daily caregiving time were more likely to be in the "low caregiving ability" class. Parents who had longer daily caregiving time had a better understanding of the treatment and care, and accumulated more caregiving experience, which helped to improve their caregiving ability. However, previous studies indicated that the care burden would increase gradually as caregivers spent more time caring for their 
children with cancer, which had a negative influence on parents' caregiving ability88Utne I, Miaskowski C, Paul SM, et al. Association between hope and burden reported by family caregivers of patients with advanced cancer. Support Care Cancer.2013; 21(9):2527-2535.. So, appropriate daily caregiving time was encouraged to help primary caregivers achieve a balance between care for their children and themselves, as a result, improving their caregiving ability.

Parents with younger children had a higher likelihood of being in the "low caregiving ability" class than in the "medium caregiving ability" and the "high caregiving ability" class. Younger children could not express their feelings and needs clearly, and they had lower self-care ability, which led to more dependence on their parents' assistance99Xie AW, Chan YY, Yao WY, et al. The investigation of nursing needs of school-aged children underwent lumbar puncture with Acute Leukemia. Chinese Nursing Management. 2018; 18(1):103107.. Parents' failure in perceiving or satisfying the needs of their children might led to parents' low selfevaluation on their caregiving ability.

In this study, parents of children with leukemia were more likely to be in the "low caregiving ability" class than in the "high caregiving ability." This is probably because $59.7 \%$ of the parents in the study had children diagnosed with leukemia whowere in the early stage (1-3 months) of treatment of leukemia.

\section{Clinical Implementation}

Exploring the latent classes of caregiving ability in parents of children with hematological malignancies and the socio-demographic characteristics of parents in the latent classes could provide useful information for the professional healthcare providers to identify parents who are most likely to have low caregiving ability. Therefore, early and precise supportive intervention could be provided to the targeted parents to improve their caregiving ability, which in results, improving the children's disease prognosis and quality of life. Moreover, the results of this study also reminded us that parents' with high self-appraisal of caregiving ability may not have high ability in all the aspects of caregiving. Therefore, targeted support needs to be provided to parents based on their needs. Additionally, parents who have high self-appraisal of caregiving ability may gradually encounter more caregiving problems and their self-appraisal of caregiving ability will change. So, healthcare providers are highly suggested to track the development of parents' caregiving ability.

\section{Limitations}

There are some limitations in the study. First, data were collected in three children's hospitals in Shanghai, Suzhou and Chongqing, China that are all big cities. Secondly, parents of children with leukemia account for majority of the sample. As such, the generalizability of our findings may be limited. Finally, after the diagnosis of their children, parents' caregiving ability changes across the children's disease treatment period. The present study is a cross-sectional study and unable to speculate the trajectory of parents' caregiving ability development. Therefore, a longitudinal study is needed to explore the developmental trend of parents' caregiving ability in the future.

\section{Conclusion}

Three distinct latent classes of parents of children with hematological malignancies were identified with regard to caregiving ability. This is the first study using LCA to examine caregiving ability in this population in China. Our findings provided useful implications for healthcare providers to better understand the classes of caregiving ability among parents of children with hematological malignancies in China, and help them provide timely supportive intervention to the parents who have low caregiving ability.

\section{Conflict of interest}

There are no potential conflicts of interest known to any of the contributing authors.

\section{Acknowledgments}

This study was supported by the National Natural Science Foundation of China (project no. 71904195), Shanghai Sailing Program from Science and Technology Commission of Shanghai Municipality, China (pro- 
ject no. 18YF1429400), and International Science and Technology Cooperation Program from Science and Technology Commission of Shanghai Municipality, China (project no. 12410707900).

\section{References}

\section{Hosted file}

Table 1.pdf available at https://authorea.com/users/361214/articles/482701-identifyinglatent-classes-of-parents-of-children-with-hematological-malignancies-with-respect-tocaregiving-ability-a-latent-class-analysis

\section{Hosted file}

Table 2.pdf available at https://authorea.com/users/361214/articles/482701-identifyinglatent-classes-of-parents-of-children-with-hematological-malignancies-with-respect-tocaregiving-ability-a-latent-class-analysis

\section{Hosted file}

Table 3.pdf available at https://authorea.com/users/361214/articles/482701-identifyinglatent-classes-of-parents-of-children-with-hematological-malignancies-with-respect-tocaregiving-ability-a-latent-class-analysis

\section{Hosted file}

Table 4.pdf available at https://authorea.com/users/361214/articles/482701-identifyinglatent-classes-of-parents-of-children-with-hematological-malignancies-with-respect-tocaregiving-ability-a-latent-class-analysis

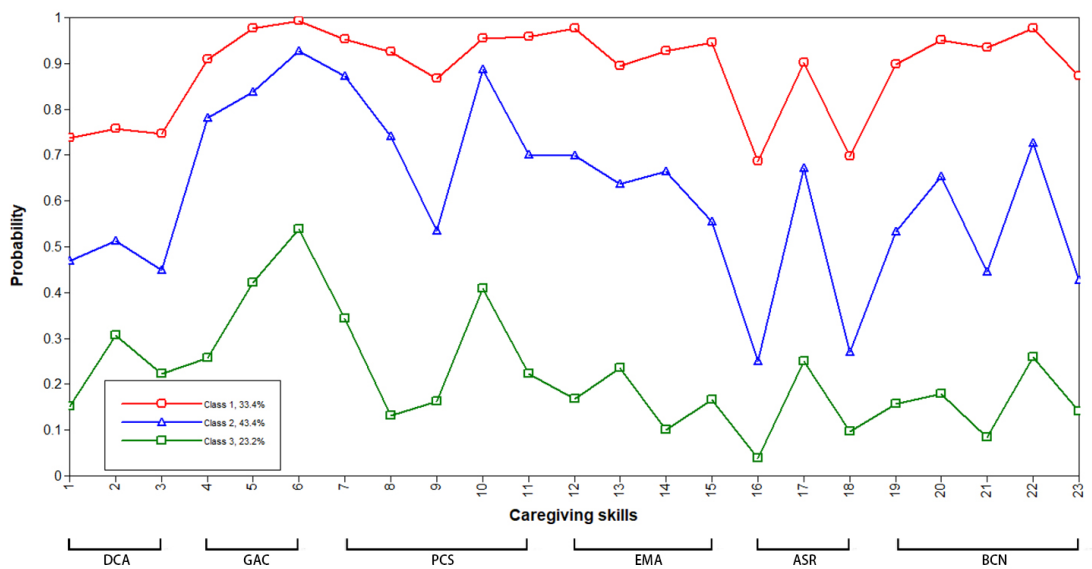

